\title{
A Unified CSF-based Framework for Edge Detection and Edge Visibility
}

\author{
Karine Joulan \\ Université Paris Est, IFSTTAR, LEPSiS \\ 58 Boulevard Lefebvre, 75015, Paris (France) \\ karine.joulandifsttar.fr
}

Roland Brémond

http://perso.lcpc.fr/roland.bremond

\begin{abstract}
One important trend in edge detection starts from current knowledge about the Human Visual System (HVS) in order to mimic some of its components. We follow this trend and propose a new edge detector which also computes the edge visibility for the HVS. Two important processes in the HVS are taken into account: visual adaptation and contrast sensitivity. Our model is in good agreement with some classical results in human vision, such as Weber's law and Ricco's law, as well as the visibility of sine and square gratings. The main contribution is to propose a unified framework, biologically inspired, which mimics human vision and computes both edge localization and edge visibility. Compared to previous approaches, the visibility of a target is estimated without segmentation of the target. This work may contribute to military applications, Intelligent Transportation Systems, and low vision simulation.
\end{abstract}

One important trend in Edge Detection started from current knowledge about the visual system in order to mimic some of its components [20]. The underlying idea was to simulate edge detection, which is achieved with great performance by biological sensors. In the following, we come back to some aspects of Marr and Hildreth's insights, and propose a new edge detector which also computes the edge visibility for the Human Visual System (HVS).

Two important processes in the HVS are taken into account in the proposed edge visibility estimation: visual adaptation and contrast sensitivity. Although the HVS cannot be described as a linear system [21, 7], it is linear in some of its components. Under this assumption, the knowledge of the systems's response to sine wave gratings allows one to predict the response to any input signal [5]. This allows to use the linear systems theory to compute the location and amplitude (visibility) of edges in natural images, providing that we compensate for visual adaptation (the main non-linear mechanism in the early steps of the HVS), which is done with a gain feedback.

In the proposed framework, Weber's law is used as a reference model for the non-linear part of the visual processing. For the linear part of the model, the Contrast Sensitivity Function (CSF) is taken as a reference vision model [2]. It describes the visibility of gratings for the HVS.

The implementation uses a set of Difference of Gaussians (DoG) operators in order to mimic the HVS. It produces outputs consistent with the CSF, when applied to the visibility of gratings. The edge localization is computed as zero-crossing of a sum of DoG, which is close to Marr and Hildreth [20] but closer to the HVS [12, 21] than Marr's Laplacian of Gaussians. The edge visibility is derived from the same sum of DoG, which results in a unified framework for the edge localization and edge visibility computation (see Fig. 1).

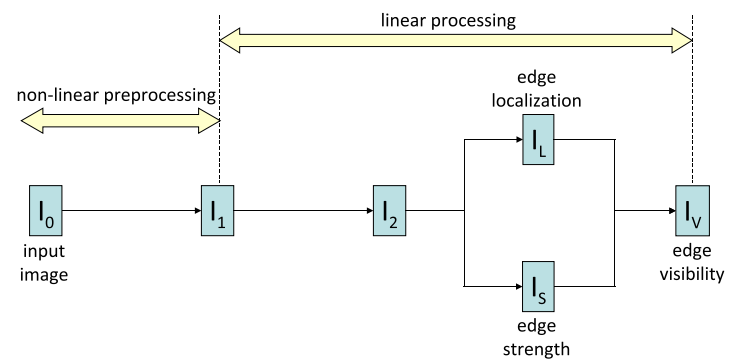

Figure 1. framework of the proposed unified model for edge detection and edge visibility computation.

Unlike most edge detectors, the purpose of the operator is to mimic the performance of the HVS, so that the usual concepts of false and true edges are revisited: a true edge is a change in intensity in the image which is detected by a human observer, even if it may be classified as "noise" in the sense that it does not separate two objects or two distinct areas of an object. With respect to Canny's idea to optimize 
edge detectors for a reference set of true and false edges, we consider true edges as visible edges, with reference to the vision science literature instead of using a priori noise description as in $[6,15]$.

This operator led to accurate results both for the edge localization and edge visibility, with respect to the visibility of gratings, and consequently with respect to the visibility of edges in natural images, under the limits of the linear system theory applied to human vision. When applied to sine gratings, our operator gives the exact solution for the edge localization and a good approximation of the edge visibility, according to psychovisual reference data [2]. Application to square gratings is also in good agreement with psychovisual data [5]. Evidence is also given that the operator's behaviour is consistent with Weber's law and Ricco's law [21]. This last result is obtained without target segmentation.

Finally, practical applications of our concept are proposed, including image quality, estimating the visibility of targets in military applications, improving existing Advanced Driver Assistance Systems (ADAS), and low vision simulation.

\section{Previous work}

The concept of edge detection is mainly considered as a first step in a bottom-up approach of Computer Vision: edges are detectable regions of objects, and their knowledge helps both to find the objects in a scene and to localize them. In this framework, edges are included in the primal sketch of the images [20], which in turn contributes to the computation of the geometric and photometric properties of objects in the scene.

Given a smoothing filter, the edges at the selected scale can be localized on the zero-crossing of its second derivative. Marr and Hildreth used the zero-crossings of the Laplacian of Gaussian $\left(\nabla^{2} G\right)$ to find the edges in an image $I$. They computed the amplitude $\nu$ of an edge from the slope $s$ of $\nabla^{2} G(I)$ on the zero-crossings: $\nu=s / 2 \pi f$, where $f$ is the frequency of the smoothing bandpass filter. However, they do not adress the issue of combining edge amplitude at various scales. It should be noted that unlike Marr's proposal, most operators derive the edge strength from the gradient's modulus on $I$.

Several classical challenges are to be addressed by any edge detector: among them, the multi-scale issue, the sensitivity to noise, and the edge strength estimation. The multi-scale problem comes with filtering. Filtering an image, most of the time, needs an implicit spatial scale, i.e. a bandwidth. Then, a multispectral edge detection is needed in order to find edges at all scales, and the multi-scale issue is to select, either the right local scale [17], or the right strategy to combine scales, fine-to-coarse [4], coarse to fine [6] or with other techniques, such as feature-based classifi- cation [22].

The sensitivity-to-noise issue looks very simple, after Canny's optimisation proposal [6]. The basic reason why false edges are detected is that an edge detector finds intensity changes in images, rather than edges of objects in the scene. Thus, some intensity changes should not be classified as object edges (for instance, it may be due to surface texture, to the sensor noise, etc.). Canny proposed an optimization approach of edge detection [6, 9, 15]: from an analytic model of false edges (stationnary additive Gaussian noise), he derived an optimal operator in a classification sense: it is the best one for the classification of true and false edges. Although limited to a specific model of true and false edges, the white noise paradigm has become a standard in the edge detection literature.

The background for this approach is that edge detectors shoud find edges in objects, in the scene, rather than intensity variations in images. In her literature review [3], Basu's point of view is closer to human vision: she states that the purpose of an edge detector is to mimic the HVS to find edges. She defines edges as intensity transitions in the images, instead of transitions between objects, in the scene [23]. Our paper follows Marr and Basu in their HVS simulation approach.

Edge strength estimation is often under-estimated in the edge detection literature, because it is not an issue in most applications of edge detection, where the challenge is to find the object's edges with the best possible accuracy. Still, most operators use two steps: the first one selects edge candidates, and the second one labels the candidates as false and true edge, based on some notion of the edge amplitude (e.g. the gradient modulus, see above) [23]. If one only wishes to detect and localize objects, the key issue is to separate "True" from "False" edges. Edge amplitude is seen, then, as a criterion for this classification. For instance, Canny proposed to improve edge detection with a hysteresis threshold on the gradient modulus, instead of a simple threshold. In some applications, however, such as visibility estimation [8, 14] and Computer Graphics [11, 1], the estimation of the edge amplitude (also called strength, intensity, visibility) becomes an issue in itself, while the "false edges" issue becomes less important (in Computer Graphics applications, the object's localization in known a priori).

Our contribution addresses edge detection as a simulation of human performance, rather than a first step in a bottom-up approach. Thus, the sensitivity-to-noise has a different meaning here, compared to the mainstream edge detection literature: it addresses the ability to mimic the HVS in its edge detection performance. The multiscale issue is addressed with a single, biologically inspired operator, the output of which is the sum of separated bandpass channels. Thus, the selection of the relevant scale is computed inside the operator, and no ambiguity is left to post- 
processing.

\section{Model}

We have merged two classical approaches from edge detection and vision science literature. First, we use a modified version of Marr and Hildreth's edge detector [20]. This new operator is closer to the HVS behaviour than Marr's operator, because it uses Difference of Gaussian operators instead of Laplacian of Gaussian. Second and more important, the edge amplitude estimates the edge visibility, in the sense of the Contrast Sensitivity Function (CSF) of the HVS $[19,2]$. Both edge localization and edge visibility are computed from a unique, biologically-inspired operator, namely a sum of Difference of Gaussian (DoG), SDoG in the following.

\subsection{True and False edges}

In most approaches, an edge detector includes 3 components: a smoothing, a derivative, and a labelling steps [23]. The derivative step being sensitive to noise, it was found sensible to remove this noise to some extend with a smoothing operator before the derivation, at the cost of lost information and spatial uncertainty. Edge detection, then, has become an art of tradeoff between these two constraints. Regularization techniques have been proposed to choose the best compromize, providing some analytical model of the false edges properties.

Most edge detector evaluations have focused so far on the detection of false edges, and after Canny's reference paper, most edge detectors used a priori knowledge of true and false edge, either to assess the detector's performance, or to build an optimal detector for a given ground truth. What is a false edge? As stated by Forsyth and Ponse (p. 166), noise is "anything we don't wish to use" [13]. Thus, we propose a new definition of false and true edges, relevant for the HVS: a false edge is an edge which is not detected by an observer in the image. We don't want to detect invisible edges. This is different from most approaches where false edges are due to the thermal noise in cameras, which is estimated with Gaussian noise.

\subsection{Visual Adaptation}

One of the first breakthrough in psychophysics history was the notion of sensorial adaptation: perception is mainly sensitive to relative variations, rather than to absolute variation. The names of Weber and Fechner are attached to this law: the ratio of the input signal to the mean signal is nearly constant, for a given sensory channel, at detection threshold. Visual adaptation can be roughly separated from other visual processing of the HVS, in order to separate linear from non-linear processing [21].

From a luminance image input, we simulate the visual adaptation with a Gain feedback, set to the inverse of the

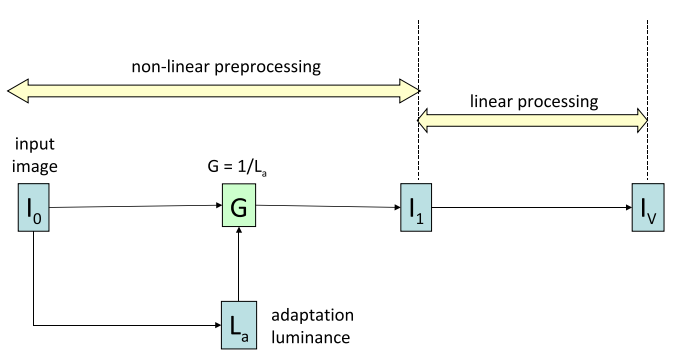

Figure 2. Simulation of the visual adaptation with a Gain feedback. A non-linear preprocessing is computed before edge detection.

adaptation luminance (see Fig. 2), that is, the inverse of the mean luminance. This pre-processing simulates Weber's law, in the sense that two edges with the same luminance contrast are set to the same intensity value after preprocessing. Thus, their computed visibility will be the same (see Fig. 1 and section 2.5).

Working with luminance images, this preliminary step is necessary; with a camera sensor and automatic gain control, it is less important. Indeed, the Gain feedback simulates visual adaptation, which happens both in biological and optical sensors.

\subsection{Contrast Sensitivity}

The Contrast Sensitivity Function (CSF) describes the visibility of sine gratings for a human observer. It is an interesting tool in the framework of linear systems, as any image may be split into gratings by Fourier decomposition. What is more, the visual system roughly behave as a linear system once the luminance adaptation is set. Thus, for instance, the sensitivity to square gratings can be predicted from the sensitivity to sine gratings [5].

In the late 70', Mannos and Sakrisson proposed a popular analytical model of the CSF to the field of Computer Science [19]. Then, Barten combined available psychovisual data to propose the most complete model of CSF to date [2]. In the following, we use Barten's CSF:

$$
C S F(f)=a f e^{-b f} \sqrt{1+c e^{b f}}
$$

where $f$ is the frequency in cycle per degree $(\mathrm{cpd}), c=$ $0.06, b=0.3(1+100 / L)^{0.15}$, and:

$$
a=\frac{540(1+0.7 / L)^{-0.2}}{1+\frac{12}{w}(1+f / 3)^{-2}}
$$

where $w$ is the stimulus size $\left(10^{\circ}\right.$ in the following), and $L$ the mean luminance. Unlike [19], this CSF depends on $L$, so that the separation between adaptation and contrast sensitivity is a rough approximation of the HVS behaviour. Hopefully, this approximation is fair enough above $30 \mathrm{~cd} / \mathrm{m}^{2}$, which includes all daylight applications. 
Another differences between the CSF proposed by Mannos and Sakrisson and Barten's model is the normalization of the CSF. In [19], the CSF is normalized, and the input image is assumed to be a sensor output, not luminance values in $\mathrm{cd} / \mathrm{m}^{2}$. If the optical sensor uses a gain modulation, just as biological sensors do, it is a clever way to address the adaptation non-linearity (section 2.2). Still, in the following, we used Barten's CSF, which is more accurate and makes it easier to compare the edge visibility to psychophysical data.

\subsection{Edge Detection}

Difference of Gaussians (DoG) was proposed in vision science as an analytic model of the receptive fields of $\mathrm{X}$ cells in the Lateral Geniculate Nucleus (LGN) in the thalamus $[12,21]$. We have implemented the CSF as a weighted sum of DoG (or SDoG), which are bandpass filters in the frequency domain. Thus, each of these DoG simulates both a bandwidth of the CSF (considering the visual system as a multiscale analyzer [5]), and ON/OFF cells with receptive fields of a given size [12].

In the following, a weighted sum of DoG will refer to:

$$
S D o G(I)=\sum_{k} \omega_{k}\left[G_{\sigma_{k}^{+}}-G_{\sigma_{k}^{-}}\right](I)
$$

where $I$ is the input image (in luminance units), $G_{\sigma}$ is the normalized Gaussian operator with Standard Deviation $\sigma$, $\sigma^{+}$and $\sigma^{-}$are the SD of the positive and negative parts of a DoG $\left(\sigma^{-}=\lambda \sigma^{+}\right)$, and $\omega$ is its weight.

A DoG is not, strictly speaking, a second derivative. However, the edge detection is achieved by a DoG with the same quality as with $\nabla^{2} G$ in terms of localization, provided that the coefficients of the positive and negative weights are the same (as in Eq. 3): the response to a step edge is null at the edge. Marr and Hildreth noticed that a $\nabla^{2} G$ is close to a DoG when $\sigma^{+}$is close to $\sigma^{-}$. Unfortunately, it is not the case in the available data: in [12], physiological measurements ranges $\lambda$ between 2.4 and 23 .

\subsection{Edge Visibility}

From the zero-crossings, most authors use the gradient modulus as an index of the edge amplitude. This index, computed on the original image $I$, may lead to good results in order to select the true edge from an over-segmented edge detection. Some problems emerge however if one uses it for edge visibility estimation. An analytic approach helps here. Suppose a 1D sine wave grating with spatial frequency $f$ :

$$
I_{f}(x)=A \sin (2 \pi f x)
$$

The gradient modulus, taken at zero-crossings, is equal to $2 \pi f A$, while the visibility of the gratings is

$$
V=A \times C S F(f)
$$

The gradient modulus is clearly a very bad estimation, as the CSF is far from linear with $f$.

Marr and Hildreth proposed that the edge strength should be proportional to the slope $s$ of the output signal $\nabla^{2} G(I)$ divided by $f$. We have followed this proposal on the SDoG, which leads to good results. Fig. 3 shows the final computational framework: the DoG contribute both to edge localization and edge visibility computation.

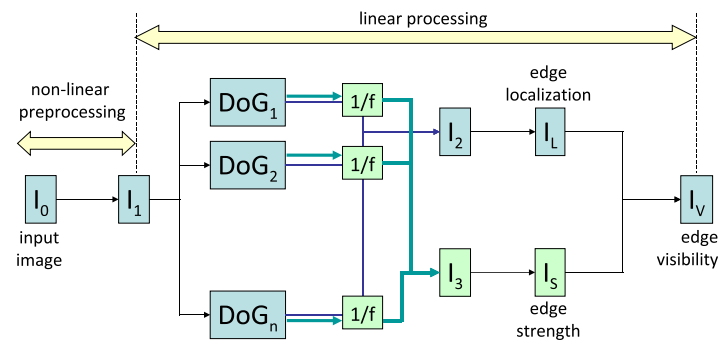

Figure 3. Framework for the edge detection and edge visibility computation, based on a CSF model.

Let's see now how to compute the set of DoG which simulates a given CSF. Using the same 1D example, the convolution of $I_{f}$ by a Gaussian function $G_{\sigma}$ is:

$$
G_{\sigma} \star I_{f}=I_{f} e^{-f^{2} \sigma^{2} / 2}
$$

Thus, the convolution with a DoG is:

$$
\left[G_{\sigma}-G_{\lambda \sigma}\right] \star I_{f}=I_{f}\left[e^{-f^{2} \sigma^{2} / 2}-e^{-f^{2} \lambda^{2} \sigma^{2} / 2}\right]
$$

The modulus of the gradient ( $s$ for slope) on the zerocrossings may be computed from:

$$
\frac{\partial}{\partial x}\left(\left[G_{\sigma}-G_{\lambda \sigma}\right] \star I_{f}\right)=2 \pi f K I_{f}
$$

with $K=e^{-f^{2} \sigma^{2} / 2}-e^{-f^{2} \lambda^{2} \sigma^{2} / 2}$, that is, $s=2 \pi A f K$. Let's consider now the gradient modulus as a function of $f$. It is maximum when $\frac{\partial s}{\partial f}=0$, which leads to

$$
\sigma=\frac{2}{f} \sqrt{\frac{\lambda^{2}-1}{\ln \lambda}}
$$

This relation between $f$ and $\sigma$ gives way to the computation of the SDoG from the CSF. The first DoG is tuned to the mode of the CSF, that is, $\sigma_{1}$ is computed from Eq. 9 where $f$ corresponds to the maximum of the CSF. Then, the weight $\omega$ is set to

$$
\omega=\frac{C S F(f)}{e^{-f^{2} \sigma^{2} / 2}-e^{-f^{2} \lambda^{2} \sigma^{2} / 2}}
$$

This procedure is repeated iteratively on each residu, for $N$ DoG ( $N=6$ in the following). Based on [12], we used 


\begin{tabular}{|l|r|r|r|r|r|r|}
\hline & DoG $_{1}$ & DoG $_{2}$ & DoG $_{3}$ & DoG $_{4}$ & DoG $_{5}$ & DoG $_{6}$ \\
\hline$f$ & 0.2 & 0.5 & 1.4 & 2.0 & 4.0 & 10.6 \\
$\sigma^{+}$ & 3.706 & 1.482 & 0.529 & 0.371 & 0.185 & 0.069 \\
$\omega$ & 11.1 & 67.1 & 202.0 & 35.5 & 620.1 & 266.1 \\
\hline
\end{tabular}

Table 1. Weigths and SD of the SDoG model $(\lambda=3)$, for Barten's CSF with $L=100 \mathrm{~cd} / \mathrm{m}^{2} . f$ and $\sigma$ are in cpd.

$\lambda=3$. Tab. 1 shows the computed values for $\sigma$ and $\omega$, from Barten's CSF [2] with $L=100$.

What is the visibility of a sine wave grating $I_{f}$ with this model? If the DoG is tuned to $f$ (thanks to Eq. 9), the slope taken on the zero crossings is:

$$
s=2 \pi f \omega A\left[e^{-f^{2} \sigma^{2} / 2}-e^{-f^{2} \lambda^{2} \sigma^{2} / 2}\right]
$$

so that

$$
V=\frac{s}{2 \pi f}=A \times C S F(f)
$$

which is the expected value. Starting from an unknown frequency $f$, the visibility is estimated by the SDoG (see Fig. 3) with:

$$
V=A \sum_{1}^{N} \omega_{k}\left[e^{-f_{k}^{2} \sigma_{k}^{2} / 2}-e^{-f_{k}^{2} \lambda^{2} \sigma_{k}^{2} / 2}\right]
$$

where $N$ is the number of DoG in the SDoG approximation, and $f_{k}$ is the frequency associated with DoG $k$ (see Eq. 9).

\section{Results}

The model was tested in several reference situations, in order to compare its predictions to those of well known vision models. These predictions concern the two aspects of the models: edge localization and edge visibility.

\subsection{Edge localization}
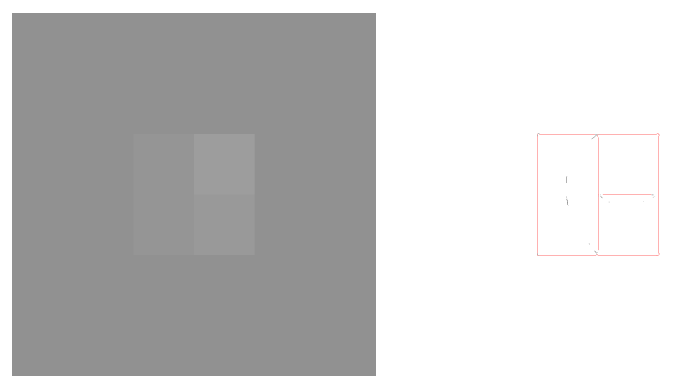

Figure 4. Left: Pattern of flat surfaces. Right: Visible edges $(V \geq$ 1 ) in red, invisible edges $(0<V<1)$ in grey levels (darker when $V$ is closer to 1 ).

Edge localization, in the case of linear step edges, is accurate as a consequence of the DoG structure. All DoG find a linear step edge with a correct localization, so do their sum. Some localization error occur at corners and junctions, as in most low level edge detectors (see Fig. 4). The main problem concerns junctions, where artifacts are created; hopefully, the visibility of these artifacts is much lower than the visibility of the "true" edges. Due to the new definition of true and false edges (section 2.1), the artifacts are classified as False edges in Fig. 4.

\subsection{Edge Visibility}

Weber's law is implicit in the model, due to the preprocessing step. Consider $I_{f, C, M}$ a sine wave grating, taken as input stimulus, with frequency $f$ (in cpd), contrast $C$ and mean luminance value $M$ (in $\mathrm{cd} / \mathrm{m}^{2}$ ). After pre-processing, $I$ becomes $I_{f, C, 1}^{\star}$, so that the visibility of the grating is not sensitive to $M$, but to $C$.

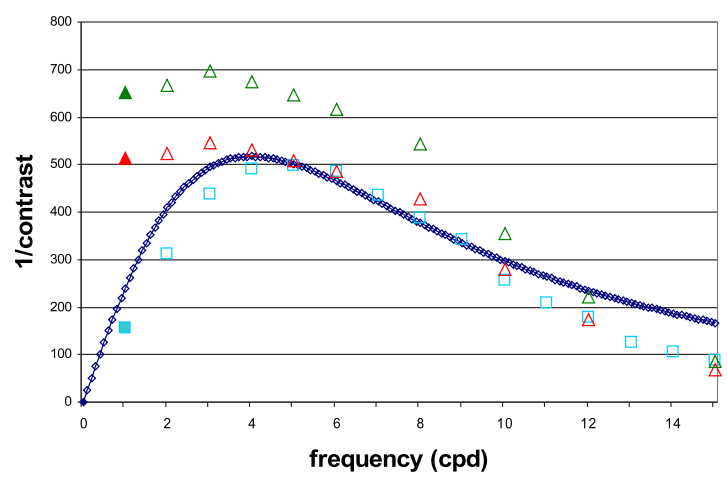

Figure 5. Dark Blue: Barten's CSF $(L=100)$. Blue: Visibility $V_{\text {sine }}$ of sine wave gratings $(M=100, C t=1)$. Green: Visibility $V_{\text {square }}$ of square gratings. Red: $4 / \pi V_{\text {square }}$.

The visibility of gratings $I_{f, 1, M}$ was compared to the $C S F$, with a good agreement, in Fig. 5. Moreover, according to [5], the visibility of sine wave and square gratings are proportional downto the CSF maximum value. This prediction was also found, and with the same ratio $r=4 / \pi$ (this factor results from the decomposition of the square grating into harmonics, see [5])).

Another effective result is that the proposed model is consistent with Ricco's law, which states that the visibility of a small target follows:

$$
\log _{10}\left(C_{s}\right)=K-\log _{10}(A)
$$

where $C_{s}$ is the visibility threshold of a disc with an area $A$. To check this, the edge visibility of discs of various sizes were computed $\left(M=100 \mathrm{~cd} / \mathrm{m}^{2}\right.$ and $C=0.01$ for all targets). If $V(r)$ is the visibility of a disc with radius $r$, its contrast level at the visibility threshold can be set to $C_{s}=$ $0.01 / V(r)$, because the visibility is linear with $C$. Thus, it was possible to plot Eq. 14 in Fig. $6\left(A=\pi r^{2}\right)$. The first 
part of the curve follows Ricco's law (linear, with slope -1), then it tends to a constant for larger targets, which is the expected behaviour.

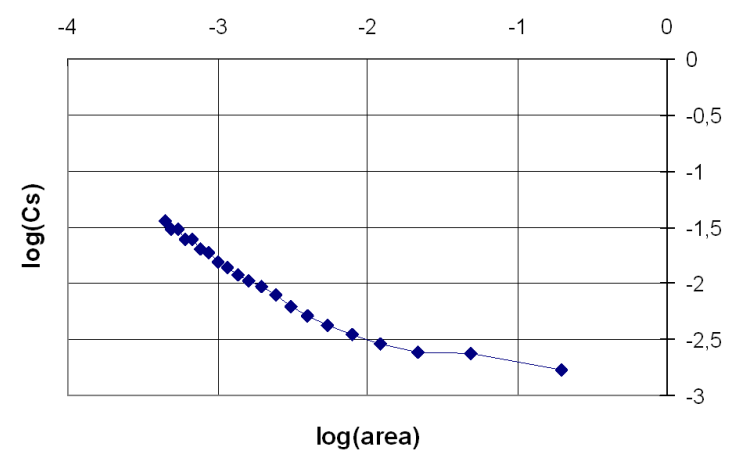

Figure 6. Estimation of Ricco's law with the proposed model: contrast threshold $C_{s}$ of discs of various angular size as a function of their area (Log-Log representation).

\section{Conclusion}

We propose a unified framework, based on a simulation of the HVS, in order to compute both the localization and visibility of edges in an image. Future work includes more intensive validation of the model against psychophysical vision models, such as contrast masking [16]. One promising improvement could be to combine zero-crossings of the SDoG, which fails to detect junctions, with local extrema, which are well known scale-invariant keypoints [10, 18].

A number of applications may benefit of this work, because it can be seen as a true human vision simulator applied to edge detection, which addresses several military concerns. Also, changing from Barten's CSF to a pathological CSF (low vision) may simulate the edge detection task for a given population. Improving existing Advanced Driver Assistance Systems (ADAS) is another possible application of our operator, as the knowledge of the driver's visibility in the scene may be used to regulate these systems.

\section{References}

[1] T. O. Aydin, M. Čadík, K. Myszkowski, and H.-P. Seidel. Visually significant edges. ACM Trans. Appl. Percept., 7:27:127:15, July 2010. 34

[2] P. G. J. Barten. Contrast Sensitivity Function and its effect on image quality. SPIE, 1999. 33, 34, 35, 37

[3] M. Basu. Gaussian-based edge-detection methods. A survey. IEEE Trans. on Systems, Man and Cybernetics, Part C, 32(3):252-260, 2002. 34

[4] F. Bergholm. Edge focusing. IEEE Trans. on Pattern Analysis and Machine Intelligence, 9:726-741, 1987. 34
[5] F. W. Campbell and J. G. Robson. Application of Fourier analysis to the visibility of gratings. Journal of Physiology, 197:551-566, 1968. 33, 34, 35, 36, 37

[6] J. Canny. A computational approach to edge detection. IEEE Trans. on Pattern Analysis and Machine Intelligence, 8:679698, 1986. 34

[7] M. Carandini, J. B. Demb, V. Mante, D. J. Tollhurst, Y. Dan, B. A. Olshausen, G. L. Gallant, and N. C. Rust. Do we know what the early visual system does? journal of Neuroscience, 25(46):10577-10597, 2005. 33

[8] S. Daly. The Visible Differences Predictor: An Algorithm for the Assessment of Image Fidelity, pages 179-206. A. B. Watson Ed., Digital Images and Human Vision, MIT Press, Cambridge, MA, 1993. 34

[9] R. Deriche. Using Canny's criteria to derive an optimal edge detector recursively implemented. International Journal on Computer Vision, 2(1), 1987. 34

[10] R. Deriche and G. Giraudon. A computational approach for corner and vertex detection. International Journal of Computer Vision, 10:101-124, 1992. 38

[11] F. Durand and J. Dorsey. Fast bilateral filtering of hdr images. In Proceedings of SIGGRAPH, pages 257-265. ACM Press, 2002. 34

[12] C. Enroth-Cugell and J. G. Robson. The contrast sensitivity of retinal ganglions cells of the cat. J. Physiol., 187:517-522, 1966. 33,36

[13] D. A. Forsyth and J. Ponse. Computer Vision: a modern approach. Prentice Hall, 2002. 35

[14] N. Hautière and D. Aubert. Visibles edges thresholding: a hvs based approach. In Proc. IEEE International Conference on Pattern Recognition (IPCR), pages 155-158, Hong-Kong (China), 2006. 34

[15] W. M. Ilhagga. The Canny edge detector revisited. International Journal of Computer Vision, 91(3):251-261, 2010. 34

[16] G. E. Legge and J. M. Foley. Contrast masking in human vision. Journal of the Optical Society of America, 70:14581470, 1980. 38

[17] T. Lindeberg. Edge detection and ridge detection with automatic scale selection. International Journal of Computer Vision, pages 117-156, 1998. 34

[18] D. G. Lowe. Distinctive image features from scale-invariant keypoints. International Journal of Computer Vision, 60(2):91-110, 2004. 38

[19] J. L. Mannos and D. J. Sakrison. The effects of a visual fidelity criterion on the encoding of images. IEEE Transactions on Information Theory, 4:525-536, 1974. 35, 36

[20] D. Marr and E. Hildreth. Theory of edge detection. In Proceedings Royal Society London B, volume 207, pages 187 217, 1980. 33, 34, 35

[21] B. Wandell. Foundations of vision. Sinauer associates, Sunderland, MA, USA, 1995. 33, 34, 35, 36

[22] D. Ziou and S. Tabbone. A multi-scale edge detector. Pattern Recognition, 26(9):1305-1314, 1993. 34

[23] D. Ziou and S. Tabbone. Edge detection technique. An overview. Journal of Pattern Recognition and Image Analysis, 8:537-559, 1998. 34, 35 